\section{Perfil clínico-epidemiológico de pacientes com doença de Chagas no Hospital Universitário de Maringá, Paraná, Brasil}

\author{
Clinical and epidemiological profile of patients \\ with Chagas disease at the University Hospital \\ in Maringá, Paraná, Brazil
}

Carlos Eduardo Bozelli 1 Silvana Marques de Araújo 2 Ana Lúcia Falavigna Guilherme 2 Mônica Lúcia Gomes 2

\section{Introdução}

This paper describes the clinical and epidemiological profile of patients with Chagas disease treated in outpatient and inpatient departments at the Maringá University Hospital (UHM) in Paraná State, Brazil, from May 1998 to May 2003. Mean age was higher among hospitalized patients $(p<0.000)$. Hospitalized patients were predominantly males, as opposed to inpatients who were mostly females ( $p=0.0033$ ). Of the 95 patients, $60 \%$ were born in the States of Minas Gerais and São Paulo and 25.3\% in Paraná. Family history for Chagas was positive in $68.9 \%$, while $53.3 \%$ reported the presence of triatomine vectors in the household. In decreasing order, the most common clinical forms were cardiac, digestive, indeterminate, and cardiodigestive. The indeterminate form predominated among outpatients, as opposed to the cardiac and digestive forms among inpatients. Chronic cardiac and digestive complications were the principal complaints at hospital admission. This study showed particularly high percentages of cardiac (38.9\%) and digestive disease (26.3\%) as compared to other geographic areas. The Maringá University Hospital provides symptomatic care for these complications and does not prioritize etiological treatment, even for patients in the indeterminate phase.

Chagas Disease; Epidemiological Profiles; University Hospitals
A doença de Chagas, que tem como agente etiológico o Trypanosoma cruzi, é atualmente descrita pela Organização Mundial da Saúde (OMS) como doença tropical em que as estratégias de controle se mostraram efetivas com a prevalência em declínio e eliminação programada 1,2 . Atualmente, a maioria dos indivíduos infectados está em fase crônica da doença que pode expressar-se sob forma indeterminada, cardíaca, digestiva e cardiodigestiva. Segundo Dias 3 , em estudo realizado em Minas Gerais, Brasil, entre os anos de 1940 e 1982, 50\% dos pacientes infectados pelo T. cruzi apresentaram manifestações clínicas e a metade deles morreu em conseqüência direta ou indireta do curso da doença. Na forma crônica indeterminada os pacientes são assintomáticos, sem alterações no eletrocardiograma e na radiografia de tórax, sendo que 2 a $5 \%$ podem evoluir anualmente para uma das formas clínicas manifestas da doença 4.

Aproximadamente 15 milhões de pessoas estão infectadas em toda a América Latina, dos quais 3,5 milhões somente no Brasil 5. No Estado do Paraná, segundo Inquérito Nacional de 1980 6, a prevalência da doença de Chagas era de $4 \%$, com estimativa de 166.511 casos positivos numa população rural de 4.164 .943 habitantes 7 . Todavia, na década de 70 na região noroeste do Paraná ocorreu uma intensa migra- 
ção dos indivíduos da zona rural para área urbana e para a Amazônia Legal 8. Em 1990 foi observado que $6,3 \%$ dos indivíduos residentes em cinco municípios dessa região apresentaram sorologia positiva para T. cruzi ${ }^{9}$, existindo assim um contingente de pacientes com doença de Chagas que necessitam ser atendidos nas unidades básicas de saúde e hospitais da região noroeste do Paraná.

Recentemente, foi publicado um volume significativo de informações sobre a prevalência de espécies vetoras do T. cruzi no Paraná e sobre o perfil epidemiológico dos indivíduos acometidos 10,11,12,13. No entanto, nenhuma informação se encontra disponível sobre a prevalência das formas clínicas da doença de Chagas nesse Estado. Além disso, se por um lado a doença de Chagas está com a eliminação programada pela OMS 2, a descentralização no controle de endemias juntamente com a programação pactuada criaram condições para que estados e municípios utilizem de forma inteligente e direcionada seus recursos financeiros. Essa forma de atuação implica o conhecimento da situação de saúde vigente. Sendo assim, o objetivo deste trabalho foi descrever o perfil clínico-epidemiológico de pacientes com doença de Chagas atendidos no Hospital Universitário de Maringá (HUM), Paraná, Brasil.

\section{Métodos}

\section{População estudada}

Foi realizado um estudo transversal, analisando 95 prontuários que corresponderam ao total de pacientes com diagnóstico de doença de Chagas atendidos no HUM no período de maio de 1998 a maio de 2003.

\section{Variáveis epidemiológicas e clínicas investigadas}

Utilizando-se um questionário, foram coletados dados referentes à idade, sexo, naturalidade, procedência, história familiar para doença de Chagas, tipo de atendimento (ambulatorial ou internação), provável mecanismo de transmissão, motivo da procura do serviço médico, formas clínicas da doença, co-morbidades, exames complementares (endoscopia digestiva alta, esofagograma, colonoscopia, enema opaco do cólon, eletrocardiograma de 12 derivações, radiografia de tórax - avaliação da área cardíaca pelo índice cardiotorácico), exames sorológicos realizados e tratamento etiológico para a doença de Chagas. Foi realizada busca ativa de dados pelo contato com os pacientes ou com os serviços de saúde para completar prontuários onde foi identificada a falta de informações consideradas imprescindíveis. Este estudo foi aprovado pelo Comitê Permanente de Ética em Pesquisa (COPEP) da Universidade Estadual de Maringá (UEM).

\section{Análise estatística}

Foi realizada análise descritiva dos dados, utilizando-se o programa Statistica 6.0 (StatSoft Inc., Tulsa, Estados Unidos). As variáveis contínuas, com distribuição normal, foram expressas também na forma de média e desvio-padrão. Para verificar a diferença entre os grupos, internados e ambulatoriais, quanto à idade $\mathrm{e}$ ao sexo dos pacientes foram utilizados os testes de " $t$ " de Student e o qui-quadrado $\left(\chi^{2}\right)$, respectivamente, com nível de significância de $5 \%$.

\section{Resultados}

Foram analisados 95 prontuários e em 20 deles (21\%) foi necessária a busca ativa para complementar os dados.

A idade dos pacientes variou de 22 a 89 anos, sendo que na faixa etária de 41 a 70 anos foram incluídos 73,7\% deles (Tabela 1). A média de idade dos pacientes atendidos no serviço de internação $(61,2$ anos $\pm 12,8)$ foi significativamente maior $(\mathrm{p}<0,000)$ que a média de idade dos pacientes atendidos no ambulatório (50,1 anos \pm $12,5)$, sendo a faixa etária maior que 70 anos a responsável por esta diferença. O sexo masculino ocorreu em $53 \%$ dos pacientes. Como pode ser observado na Tabela 1 , o sexo masculino predominou entre os pacientes internados e o feminino entre os ambulatoriais $(p=0,0033)$.

Quanto à naturalidade, 34 (35,8\%) pacientes nasceram no Estado de Minas Gerais, 24 (25,3\%) no Paraná, 23 (24,2\%) em São Paulo, seis $(6,3 \%)$ na Bahia, cinco $(5,3 \%)$ em Pernambuco, um (1,1\%) em Alagoas, um (1,1\%) em Mato Grosso do Sul e um $(1,1 \%)$ na Paraíba.

A Figura 1 mostra os municípios de procedência dos pacientes com doença de Chagas atendidos no HUM. A maioria dos pacientes (58; $61,1 \%)$ residia no Município de Maringá e 37 (38,9\%) em cidades circunvizinhas, todas na região noroeste do Paraná. Oito $(8,4 \%)$ eram procedentes de Sarandi, quatro $(4,2 \%)$ de Nova Esperança, quatro $(4,2 \%)$ de Doutor Camargo, quatro (4,2\%) de Paiçandu. Os 17 pacientes restantes procederam de Astorga, Atalaia, Barbosa Ferraz, Campo Mourão, Colorado, Floraí, Floresta, Iguaraçu, Itaguajé, Itambé, Munhoz de 
Faixa etária e sexo dos pacientes com doença de Chagas de acordo com o tipo de atendimento no Hospital Universitário de Maringá, Maringá, Paraná, Brasil, de maio de 1998 a maio de 2003.

\begin{tabular}{|c|c|c|c|c|c|c|c|}
\hline \multirow{2}{*}{$\begin{array}{l}\text { Faixa etária } \\
\text { (anos) }\end{array}$} & \multicolumn{6}{|c|}{ Sexo } & \multirow[t]{2}{*}{ Total (\%) } \\
\hline & $\%$ & $\begin{array}{l}\text { Feminino } \\
\text { Internado }\end{array}$ & Ambulatorial & $\%$ & $\begin{array}{l}\text { Masculino } \\
\text { Internado }\end{array}$ & Ambulatorial & \\
\hline $20-30$ & 4,4 & 0 & 2 & 2,0 & 0 & 1 & 3,2 \\
\hline $31-40$ & 4,4 & 0 & 2 & 12,0 & 4 & 2 & 8,4 \\
\hline $41-50$ & 24,4 & 2 & 9 & 18,0 & 7 & 2 & 21,1 \\
\hline $51-60$ & 31,1 & 8 & 7 & 24,0 & 5 & 7 & 27,4 \\
\hline $61-70$ & 20,0 & 4 & 5 & 28,0 & 12 & 2 & 24,2 \\
\hline Mais que 70 & 15,6 & 5 & 1 & 16,0 & 8 & 0 & 15,8 \\
\hline Total & 100,0 & 19 & $26^{*}$ & 100,0 & $36^{*}$ & 14 & 100,0 \\
\hline
\end{tabular}

* $p=0,0033$.

Melo, Mandaguaçu, Marialva, Ourizona, Santa Fé, Umuarama e Uniflor.

Para 61 pacientes foi possível obter a história familiar para doença de Chagas. Destes, 42 $(68,9 \%)$ tiveram história familiar positiva e 19 $(31,1 \%)$ referiram desconhecer a história dessa doença na família.

Em 30 (31,6\%) prontuários constavam informações que permitiam relacionar com o mecanismo de transmissão. Dezesseis pacientes $(53,3 \%)$ relataram a presença de triatomíneos no domicílio, um $(3,3 \%)$ referiu ter recebido transfusão sangüínea pregressa; no entanto, 13 (43,3\%) desconheciam o vetor e não tinham recebido transfusão de sangue.

A insuficiência cardíaca congestiva (ICC), a sorologia positiva para doença de Chagas e obstipação intestinal foram os principais motivos que levaram os pacientes a procurar atendimento no HUM. Para os pacientes atendidos no ambulatório, a sorologia positiva e os sintomas de arritmia cardíaca foram os motivos mais freqüentes, ao passo que para os internados foram a obstipação intestinal e sintomas de ICC (Tabela 2).

A forma clínica cardíaca foi a de maior ocorrência, seguida pelas formas digestiva, indeterminada e cardiodigestiva (Tabela 3). Para os pacientes nascidos no Estado do Paraná, a forma clínica indeterminada foi a mais freqüente $(11 / 24)$, seguida pela digestiva $(8 / 24)$, cardíaca $(4 / 24)$ e cardiodigestiva $(1 / 24)$. A forma indeterminada prevaleceu entre os pacientes atendidos no ambulatório e as formas cardíaca e digestiva ocorreram igualmente entre os pacientes internados.

Em 54 (56,8\%) pacientes foi identificada a presença de co-morbidades. As principais doen- ças associadas foram: hipertensão arterial sistêmica, diabetes mellitus, doença pulmonar obstrutiva crônica e doença arterial coronária (Tabela 4), sendo que esta última ocorreu somente em pacientes com a forma digestiva da doença de Chagas.

Em todos os prontuários constava a informação de que os pacientes apresentavam sorologia positiva para doença de Chagas. Em $28,4 \%$ deles constavam que o método sorológico utilizado para o diagnóstico foi a imunofluorescência indireta, em $28,4 \%$ a enzimaimunoensaio (ELISA) e em $7,4 \%$ a hemaglutinação indireta. Em 34 (35,8\%) o método utilizado não foi discriminado.

Em 39 pacientes que relataram queixas digestivas foram realizados exames complementares específicos, incluindo endoscopia digestiva alta em 16 (41\%), esofagograma em 17 (44\%), colonoscopia em 6 (15\%) e enema opaco em 29 (74\%). Foi identificada a presença de comprometimento do cólon em 20 pacientes (51,3\%), e do esôfago em 10 (25,6\%) e associação de ambos em 9 (23,1\%).

Do total de pacientes atendidos no HUM, o eletrocardiograma foi normal em $44(46,3 \%)$ pacientes. Os demais apresentaram alterações eletrocardiográficas, principalmente o bloqueio completo de ramo direito associado ao bloqueio da divisão ântero-superior esquerda, o ritmo de marca-passo artificial e a arritmia ventricular (Tabela 5).

De 51 pacientes com comprometimento cardíaco, a radiografia de tórax identificou a presença de cardiomegalia em $64,7 \%$ dos cardiopatas com doença de Chagas, representando $34,7 \%$ dos pacientes com esta doença atendidos no HUM. 
Municípios de procedência dos pacientes com doença de Chagas atendidos no Hospital Universitário de Maringá, Maringá, Paraná, Brasil.
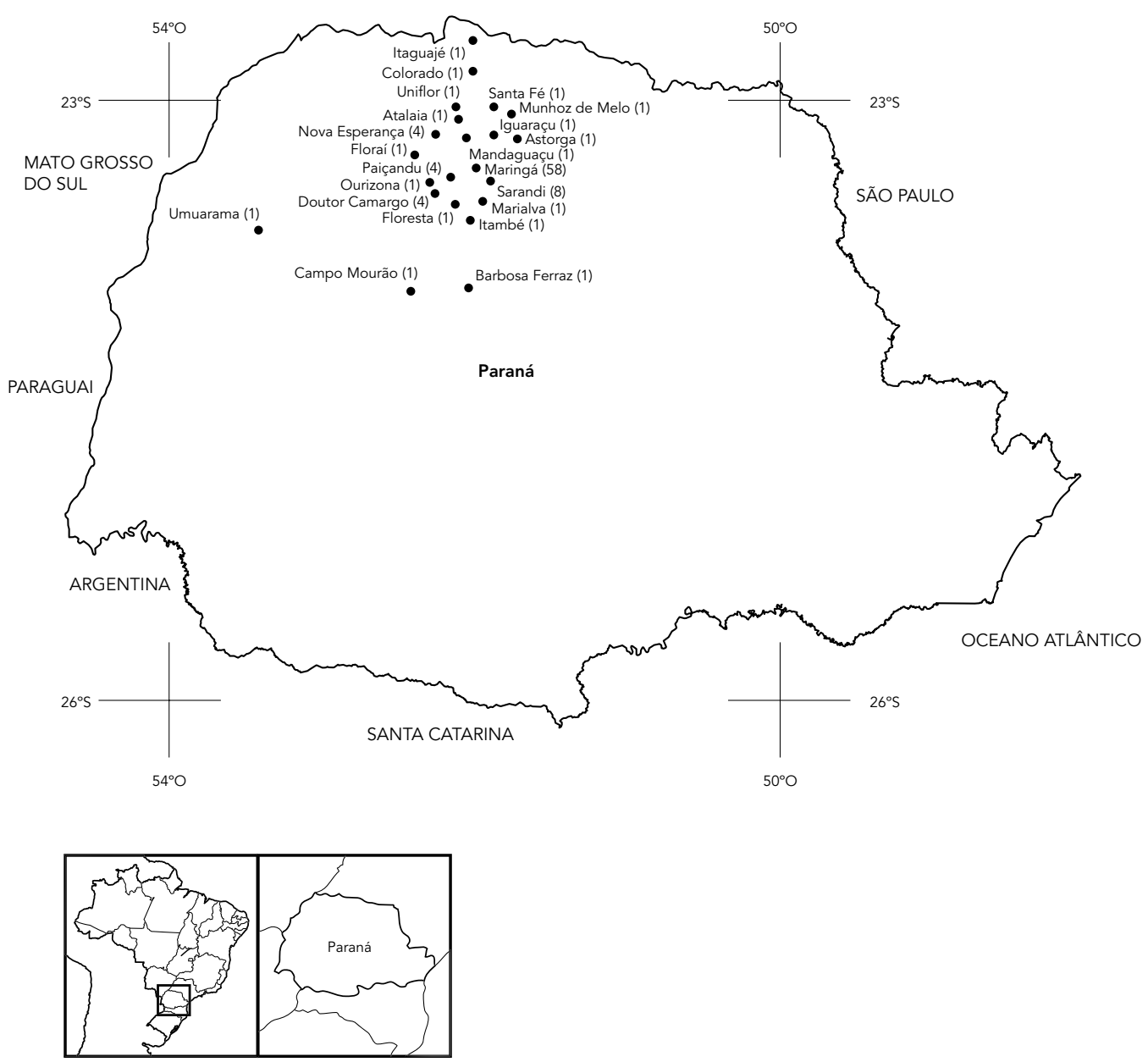

Nota: o número entre parênteses significa o número de pacientes de cada município.

O tratamento etiológico para doença de Chagas foi referido para $12(12,6 \%)$ pacientes, sendo que sete apresentaram a forma indeterminada da doença, três a cardíaca, um a digestiva e um a cardiodigestiva. Para $48(50,5 \%)$, este não foi realizado e para $35(36,8 \%)$ não foi possível obter tal dado.

\section{Discussão}

Neste trabalho foi relatado o perfil clínico-epidemiológico dos pacientes com doença de Chagas atendidos no HUM. Cabe destacar que esse hospital é centro de referência para o noroeste do Paraná e no período deste estudo atendeu pacientes com doença de Chagas procedentes de 22 municípios dessa região. Segundo Gontijo et al. 14 e Ronan et al. 15, esses pacientes são onerosos ao sistema público de saúde e indesejáveis ao setor privado. O Ministério da Saúde do Brasil preconiza que os serviços públicos devem ser de qualidade e com baixos custos. Sendo assim, os hospitais universitários devem proporcionar atendimento diferenciado aos pacientes com doença de Chagas no intuito de prevenir as complicações da doença reduzindo os custos.

Os resultados referentes ao sexo e idade dos pacientes ambulatoriais atendidos no HUM di- 
Motivos da procura de assistência médica dos pacientes com doença de Chagas atendidos no Hospital Universitário de Maringá, Maringá, Paraná, Brasil, de maio de 1998 a maio de 2003.

\begin{tabular}{|c|c|c|c|c|c|c|}
\hline \multirow[t]{2}{*}{ Motivos } & \multicolumn{2}{|c|}{ Ambulatório } & \multicolumn{2}{|c|}{ Internação } & \multicolumn{2}{|c|}{ Total } \\
\hline & $\mathrm{n}$ & $\%$ & $\mathrm{n}$ & $\%$ & $\mathrm{n}$ & $\%$ \\
\hline Sintomas de insuficiência cardíaca congestiva & 6 & 15,0 & 15 & 27,3 & 21 & 22,1 \\
\hline Sorologia positiva & 20 & 50,0 & 0 & 0,0 & 20 & 21,1 \\
\hline Obstipação intestinal & 2 & 5,0 & 17 & 30,9 & 19 & 20,0 \\
\hline Sintomas de arritmia cardíaca & 8 & 20,0 & 5 & 9,1 & 13 & 13,7 \\
\hline Disfagia & 3 & 7,5 & 9 & 16,4 & 12 & 12,6 \\
\hline Oclusão intestinal & 0 & 0,0 & 4 & 7,3 & 4 & 4,2 \\
\hline Acidente vascular cerebral & 1 & 2,5 & 1 & 1,8 & 2 & 2,1 \\
\hline Obstipação intestinal e disfagia & 0 & 0,0 & 2 & 3,6 & 2 & 2,1 \\
\hline $\begin{array}{l}\text { Obstipação intestinal e insuficiência } \\
\text { cardíaca congestiva }\end{array}$ & 0 & 0,0 & 2 & 3,6 & 2 & 2,1 \\
\hline Total & 40 & 100,0 & 55 & 100,0 & 95 & 100,0 \\
\hline
\end{tabular}

ferem de outros autores $15,16,17,18$ que atenderam no ambulatório pacientes do sexo masculino, com menor idade e oriundos de bancos de sangue. Já os pacientes atendidos no serviço de internação apresentaram média de idade significativamente maior do que daqueles atendidos no ambulatório. Esta diferença pode estar relacionada ao motivo da procura pelos serviços. Ao ambulatório recorreram predominantemente pacientes na forma indeterminada da doença de Chagas, o que está de acordo com outros autores $16,17,18$, enquanto que no setor de internação as formas cardíaca e digestiva prevaleceram, sendo a obstipação intestinal a principal queixa. Isto pode ser o reflexo da ausência de intervenções preventivas, incluindo o tratamento etiológico, que poderiam amenizar esses quadros 19. A presença dessas complicações em pacientes internados eleva os custos terapêuticos como relatado por França \& Abreu 20 e Dias 21, e pode também estar relacionada ao fato desses pacientes apresentarem maior tempo de evolução da doença ${ }^{22}$. Estes dados ressaltam a importância dos hospitais universitários no atendimento aos pacientes com doença de Chagas, uma vez que, de acordo com França \& Abreu 20, em 1996, 27,3\% dos pacientes chagásicos do país eram atendidos nesses hospitais.

Pela primeira vez é relatada a distribuição de formas clínicas para as regiões norte e noroeste do Estado do Paraná, sendo a forma cardíaca a mais freqüente entre os pacientes aqui investigados, com percentual de cardiomegalia superior à observada por outros autores 12,13,17. Para pacientes com queixas digestivas, o per-
Tabela 3

Forma clínica da doença de Chagas dos pacientes atendidos no Hospital Universitário de Maringá, Maringá, Paraná, Brasil, de maio de 1998 a maio de 2003.

\begin{tabular}{lrrrrrr}
\hline \multirow{2}{*}{ Forma clínica } & \multicolumn{2}{c}{ Ambulatorial } & \multicolumn{2}{c}{ Internados } & \multicolumn{2}{c}{ Total } \\
& $\mathrm{n}$ & $\%$ & $\mathrm{n}$ & $\%$ & $\mathrm{n}$ & $\%$ \\
\hline Indeterminada & 19 & 47,5 & 0 & 0,0 & 19 & 20,0 \\
Cardíaca & 15 & 37,5 & 22 & 40,0 & 37 & 38,9 \\
Digestiva & 3 & 7,5 & 22 & 40,0 & 25 & 26,3 \\
Cardiodigestiva & 3 & 3,75 & 11 & 20,0 & 14 & 14,7 \\
Total & 40 & 100,0 & 55 & 100,0 & 95 & 100,0 \\
\hline
\end{tabular}

centual de "megavísceras" foi também mais elevado do que o observado por Souza \& Guariento 16 , destacando-se um percentual importante da forma digestiva para os pacientes oriundos do Estado do Paraná. Considerando que a localização geográfica e a cepa de T. cruzi podem influenciar a patogenia da doença de Chagas 23 , estes achados juntamente com o encontro do T. cruzi II no Paraná 24 abrem perspectivas para que futuros estudos explorem melhor estas relações.

As principais doenças associadas identificadas em pacientes atendidos no HUM foram hipertensão arterial sistêmica e diabetes mellitus, em concordância com outros autores 15,16, 25,26. Em 12,6\% dos pacientes a doença associada foi a causa da procura de assistência médica, evidenciando que nem sempre o paciente recorre ao serviço de saúde por apresentar doença de Chagas. 
Tabela 4

Pacientes com doença de Chagas atendidos no Hospital Universitário de Maringá, Maringá, Paraná, Brasil, com ou sem a presença de doenças associadas, de maio de 1998 a maio de 2003.

\begin{tabular}{lrr}
\hline Co-morbidades & $\mathbf{n}$ & $\%$ \\
\hline Ausência de doenças associadas & 41 & 43,1 \\
Hipertensão arterial sistêmica & 24 & 25,3 \\
Diabetes mellitus & 10 & 10,5 \\
Doença pulmonar obstrutiva crônica & 4 & 4,2 \\
Doença arterial coronária & 3 & 3,2 \\
Hanseníase & 2 & 2,1 \\
Doença de Parkinson & 2 & 2,1 \\
Epilepsia & 2 & 2,1 \\
Hepatite viral crônica & 2 & 2,1 \\
Tuberculose pulmonar & 1 & 1,1 \\
Trombose venosa profunda & 1 & 1,1 \\
Síndrome nefrótica & 1 & 1,1 \\
Artrite reumatóide & 1 & 1,1 \\
Hemofilia A & 1 & 1,1 \\
Total & 95 & 100,0
\end{tabular}

\section{Tabela 5}

Resultados eletrocardiográficos dos pacientes com doença de Chagas atendidos no Hospital Universitário de Maringá, Maringá, Paraná, Brasil, de maio de 1998 a maio de 2003.

\begin{tabular}{lrr}
\hline Resultados & $\mathbf{n}$ & $\%$ \\
\hline Normal & 44 & 46,3 \\
Bloqueio completo do ramo direito associado & 21 & 22,1 \\
ao bloqueio da divisão ântero-superior esquerda & 12 & 12,6 \\
Ritmo de marca-passo artificial & 8 & 8,4 \\
Arritmia ventricular & 4 & 4,2 \\
Bloqueio completo do ramo direito & 4 & 4,2 \\
Bloqueio da divisão ântero-superior esquerda & 1 & 1,1 \\
Área eletricamente inativa ântero-septal & 1 & 1,1 \\
Bloqueio completo do ramo esquerdo & 95 & 100,0 \\
Total & & \\
\hline
\end{tabular}

Apenas sete dos 19 pacientes na forma indeterminada receberam o tratamento etiológico, apesar de ainda serem incluídos em faixa etária produtiva (média de 45,9 anos). Embora vários autores 19,27,28,29 relatem que pacientes com doença de Chagas tratados etiologicamente não evoluem na sua forma clínica ou evoluem de forma mais favorável em relação aos não-tratados, esta conduta parece não ser uma prática freqüente entre os profissionais médi- cos dessa região. Neste enfoque, é oportuno considerar que alternativas de atendimento aos pacientes com doença de Chagas como o apoio psicossocial pode atuar de forma preventiva, já que pacientes sintomáticos apresentam mais estresse físico e psicológico, menor capacidade de resiliência expressa por maior desesperança e dificuldades emocionais, menor capacidade de tenacidade e inovação 30 . O Programa ACHEI, desenvolvido pela UEM, mesmo sem a integração com o HUM, mostrou na prática o benefício desse tipo de abordagem 13. Trabalhos recentes vêm demonstrando a importância de se considerar a relação emoção/infecção na evolução de inúmeras patologias 31,32,33,34. Dentro de uma perspectiva educativa, hospitais-escola deveriam ter o compromisso ético de explorar essas possibilidades.

A história positiva para doença de Chagas nas famílias dos pacientes que apresentavam essa doença e foram atendidos no HUM, está diretamente associada com a predominância de respostas compatíveis com a hipótese de transmissão vetorial que foi observada para $53 \%$ dos pacientes aqui analisados. Estes dados estão de acordo com Gontijo et al. 17, embora um percentual muito mais elevado (95\% a 98\%) para essa forma de transmissão tenha sido reportada por estes e por outros autores 12,13. Essa diferença pode estar relacionada a falhas no preenchimento dos prontuários médicos do HUM. Estes dados, juntamente com outros aqui relatados mostram que mesmo em um hospital-escola, de referência regional, há deficiência no registro de informações.

Os resultados apresentados neste trabalho indicam que ao HUM recorrem tanto pacientes com doença de Chagas sem sintomas, quanto com complicações crônicas dessa doença. $\mathrm{O}$ conhecimento do perfil clínico-epidemiológico dos pacientes com doença de Chagas que se dirigem ao HUM permitirá a melhoria no atendimento, desde que condutas sistematizadas, elaboração de protocolos com a finalidade de trabalho preventivo, grupos de apoio psicossocial integrado ao tratamento clínico, terapêutico ou ambos sejam implantados. Fazem parte dessas estratégias a capacitação de profissionais de saúde, principalmente nas unidades básicas de saúde e no Programa Saúde da Família, e a utilização dos recursos financeiros de forma racional e direcionada. O HUM deveria ainda atuar diretamente na capacitação e formação de profissionais de saúde visando a incrementar a coleta e o registro da história clínica dos pacientes atendidos, bem como o seguimento de cada um deles. 


\section{Resumo}

Este trabalho descreveu o perfil clínico-epidemiológico de pacientes com doença de Chagas atendidos no ambulatório e na internação do Hospital Universitário de Maringá (HUM), Paraná, Brasil, entre maio de 1998 a maio de 2003. A média de idade foi maior no serviço de internação $(p<0,000)$. O sexo masculino predominou entre os internados e ofeminino entre os ambulatoriais ( $p=0,0033)$. De 95 pacientes, 60\% nasceram em Minas Gerais e São Paulo e 25,3\% no Paraná. A história familiar para doença de Chagas foi positiva em $68,9 \%$ deles e 53,3\% relataram a presença de triatomíneos no domicílio. Em ordem decrescente ocorreram as formas clínicas cardíaca, digestiva, indeterminada e cardiodigestiva. A forma indeterminada prevaleceu entre os ambulatoriais e as formas cardíaca e digestiva entre os internados. As complicações crônicas cardiacas e digestivas foram as principais queixas para a internação. Destacam-se os altos percentuais de doença cardíaca (38,9\%) e digestiva (26,3\%) encontrados neste estudo, de forma distinta ao que acontece em outras regiões geográficas. O HUM disponibiliza o tratamento sintomático dessas complicações e não prioriza o tratamento etiológico mesmo para pacientes em fase indeterminada.

Doença de Chagas; Perfis Epidemiológicos; Hospitais Universitários

\section{Referências}

1. Chagas C. Nova entidade mórbida do homem. Resumo geral dos estudos etiológicos e clínicos. Mem Inst Oswaldo Cruz 1911; 3:219-75.

2. World Health Organization. Control of Chagas disease. http://www.who.int/ctd/chagas/index. htm (acessado em 15/Fev/2005).

3. Dias JCP. Doença de Chagas em Bambuí, Minas Gerais, Brasil. Estudo clínico epidemiológico a partir da fase aguda entre 1940 e 1982 [Tese de Doutorado]. Belo Horizonte: Faculdade de Medicina, Universidade Federal de Minas Gerais; 1982.

4. Dias JCP. The indeterminate form of human chronic Chagas disease. A clinical epidemiological review. Rev Soc Bras Med Trop 1989; 22:147-56.

5. World Health Organization. Control of Chagas disease. Geneva: World Health Organization; 2002. (Second Techincal Report Series, 905).

6. Camargo ME, Silva GR, Castilho EA, Silveira AC. Inquérito sorológico da prevalência de infecção chagásica no Brasil, 1975-1980. Rev Inst Med Trop São Paulo 1984; 26:192-204.

7. Silveira AC, De Rezende FD. Epidemiologia e controle da transmissão vetorial da doença de Chagas no Brasil. Rev Soc Bras Med Trop 1994; 27 Suppl III:11-22.

8. Alegre M, Moro DA. A mobilidade da população nas antigas áreas cafeeiras do norte do Paraná. Boletim de Geografia, Maringá 1986; 4:28-73.

\section{Colaboradores}

C. E. Bozelli realizou a coleta e análise dos dados, o levantamento bibliográfico e a redação do trabalho. M. L. Gomes participou no delineamento do estudo, análise e discussão dos resultados e da redação do trabalho. S. M. Araújo auxiliou no delineamento do estudo, na discussão dos resultados e revisão crítica final do artigo. A. L. F. Guilherme colaborou na análise dos dados e revisão crítica final do artigo.

\section{Agradecimentos}

A Sebastião Gazolla pelo auxílio na análise estatística do artigo.

9. Gomes ML, Bertolini DA, Silveira TGV, Lonardoni MV, Arraes SMAA. Investigação sorológica da doença de Chagas e isolamento do Trypanosoma cruzi em indivíduos de cinco municípios da região noroeste do Paraná. Rev Soc Bras Med Trop 1992; 25 Suppl III:94.

10. Falavigna-Guilherme AL, Pavanelli GC, Silva SV, Costa AL, Araújo SM. Secondary triatomine species in dwellings and other nearby structures in municipalities under epidemiological surveillance in the State of Paraná, Brazil. Rev Panam Salud Pública 2001; 9:385-92.

11. Falavigna-Guilherme AL, Santana R, Pavanelli GC, Lorosa ES, Araújo SM. Triatomine infestation and vector-borne transmission of Chagas disease in northwest and central Paraná, Brazil. Cad Saúde Pública 2004; 20:1191-200.

12. Gomes ML, Ramos M, Zanzarini PD, Galvão LMC, Chiari E, Araújo SM, et al. Clinical, laboratory and epidemiological data of chronic chagasic patients residing in the northwestern region of the State of Paraná, Brazil. Acta Sci 2000; 22:587-92.

13. Araújo SM, Andó MH, Cassarotti DJ, Mota DCGD, Borges SMR, Gomes ML. Programa ACHEI: atenção ao chagásico com educação integral no Município de Maringá e Região Noroeste do Paraná, Brasil. Rev Soc Bras Med Trop 2000; 33:565-72.

14. Gontijo ED, Castilho M, Bonomi D, Jannuzzi JH, 
Vitor E. Estudo da evolução da cardiopatia chagásica em pacientes atendidos no ambulatório de doença de chagas do HCUFMG, no período de 1985 a 2000. In: Anais da XVI Reunião Anual de Pesquisa Aplicada em Doença de Chagas. Uberaba: Sociedade Brasileira de Medicina Tropical; 2000. p. 92.

15. Ronan L, Souza LRMF, Freitas GL, Ward LS, Almeida EA, Wanderley JS, et al. Evolução de pacientes chagásicos acompanhados em um serviço de referência. Rev Soc Bras Med Trop 2000; 33 Suppl II:91.

16. Souza LRMF, Guariento ME. Evolução de pacientes chagásicos acompanhados em um serviço de referência. Rev Soc Bras Med Trop 1998; 31 Suppl III:54-5.

17. Gontijo ED, Rocha MOC, Oliveira UT. Perfil clínico-epidemiológico de chagásicos atendidos em ambulatório de referência e proposição de modelo de atenção ao chagásico na perspectiva do SUS. Rev Soc Bras Med Trop1996; 2:101-8.

18. Lorenzi AL, Almeida EA, Cunha KAS, Camilo MVRFC. Triagem da doença de Chagas do hospital de clínicas da UNICAMP: perfil da população. In: Anais da XVI Reunião de Pesquisa Aplicada em Doença de Chagas. Uberaba: Sociedade Brasileira de Medicina Tropical; 2000. p. 90.

19. Fragata Filho AA, Boianain E, Silva MAD, Correia EB, Borges Filho R, Martins C, et al. Validade do tratamento etiológico da fase crônica da doença de Chagas com benznidazol. Arq Bras Cardiol 1995; 65 Suppl I:71.

20. França SB, Abreu DMX. Morbidade hospitalar por doença de Chagas no Brasil. Rev Soc Bras Med Trop 1996; 29:109-15.

21. Dias JCP. Control of Chagas' disease in Brazil. Parasitol Today 1987; 3:336-41.

22. Silva AL. Chagas disease surgery. Mem Inst Oswaldo Cruz 1999; 94:343-7.

23. Pereira JB, Coura JR. Diferenças regionais na doença de Chagas. Aspectos clínicos, epidemiológicos e laboratoriais de duas áreas endêmicas do Brasil. Rev Soc Bras Med Trop 1993; 28 Suppl 2:70-2.
24. Zalloum L, Gomes ML, Kinoshita AT, Toledo MJO, Prioli AJ, Araújo SM. Trypanosoma cruzi: two genetic groups in Paraná State, Southern Brazil. Exp Parasitol 2005; 111:55-8.

25. Santos VM, Cunha SFC, Teixeira VPA, Monteiro JP, Santos JAM, Santos TAM, et al. Freqüência de diabetes mellitus e hiperglicemia em mulheres chagásicas e não-chagásicas. Rev Soc Bras Med Trop 1999; 32:489-96.

26. Rocha A, Oliveira LCM, Alves RS, Lopes ER. Despopulação neuronal pancreática em chagásicos crônicos. Rev Soc Bras Med Trop 1998; 31:43-9.

27. Viotti R, Vigliano C, Armenti H, Segura E. Treatment of chronic Chagas disease with benznidazole: clinical and serologic evolution of patients with long-term follow-up. Am Heart J 1994; 127:151-62.

28. Cançado JR. Long term evaluation of etiological treatment of Chagas disease with benznidazole. Rev Inst Med Trop São Paulo 2002; 44:29-37.

29. Coura JR, Castro SL. A critical review on chagas disease chemotherapy. Mem Inst Oswaldo Cruz 2002; 97:3-24.

30. Mota DA, Gomes ML, Pereira AM, Araújo S. Estresse e resiliência em doença de Chagas. Rev Soc Bras Med Trop 2005; 38 Suppl I:332.

31. Leserman J, Petitto JM, Gu H, Gaynes BN, Barroso J, Golden RN, et al. Progression to AIDS, a clinical AIDS condition and mortality: psychosocial and physiological predictors. Psychol Med 2002; 32: 1059-73.

32. Cohen S, Doyle WJ, Turner RB, Alper CM, Skoner DP. Emotional style and susceptibility to the common cold. Psychosom Med 2003; 65:652-7.

33. Kopnisky KL, Stoff DM, Rausch DM. Workshop report: the effects of psychological variables on the progression of HIV-1 disease. Brain Behav Immun 2004; 18:246-61.

34. Petrie KJ, Fontanilla I, Thomas MG, Booth RJ, Pennebaker JW. Effect of written emotional expression on immune function in patients with human immunodeficiency virus infection: a randomized trial. Psychosom Med 2004; 66:272-5.

Recebido em 15/Jun/2005

Versão final reapresentada em 30/Set/2005 Aprovado em 07/Nov/2005 